\title{
Low-Energy Scanning Electron Transmission Measurements of Thin Polymer Films Employed in Organic Solar Cells
}

\author{
M. Pfaff*, E. Müller*, M.F.G. Klein**, A. Colsmann**, U. Lemmer** and D. Gerthsen* \\ * Laboratory for Electron Microscopy and Center for Functional Nanostructures (CFN), Karlsruhe \\ Institute of Technology (KIT), 76128 Karlsruhe, Germany \\ ** Light Technology Institute, Karlsruhe Institute of Technology (KIT), 76128 Karlsruhe, Germany
}

Low-energy scanning transmission electron microscopy (STEM) is applied to image and quantify thickness and composition of irradiation-sensitive polymer films for solar cell applications. The experiments were carried out with a dual-beam FEI Strata400S state of art microscope. A standard semiconductor STEM detector with several ringlike segments was used which is positioned below the polymer samples. High-angle annular dark-field (HAADF) images originating from electrons transmitted in a hollow cone between 0.2-0.7 rad show composition-sensitive contrast [1] which is more pronounced at low energies. Low electron energies are also preferable for the investigation of materials sensitive to knock-on damage.

Thin films of semiconducting polymers employed in organic solar cells are examined in this work. The research focuses on the morphology of the absorber layer, which contains a blend of the fullerene derivate PCBM ([6,6]-phenyl C61-butyric acid methyl ester) as acceptor and the donor material P3HT (poly(3hexylthiophen). The degree of decomposition of these two materials is important for exciton separation and charge transport to the electrodes.

Monte Carlo (MC) simulations performed by the NISTMonte-package [2] are carried out to distinguish contrast induced by thickness or composition variations and to quantify the experimental data. The MC simulations are based on Mott cross sections to model elastic scattering and the energy loss formula of Joy and Luo [3].

As seen in Fig. 1.a. the simulated fraction of the transmitted electrons shows a maximum for a specific specimen thickness which depends also on the electron energy (Fig. 1.b). This characteristic feature allows precise determination of film thickness by measuring the transmitted intensity for different electron energies. For the purpose of validation, FIB (focused-ion-beam) sectioning is used to cut crosssectional trenches into the sample to measure the film thickness. A modified semi-empirical equation following the theory of Bothe [4] can be fitted to describe the transmitted intensity at the maximum showing a good agreement for materials with low atomic number and low energies of the electron probe (Fig. 2.a.).

Monte Carlo simulations (Fig. 1) also yield information about the optimum electron energy for imaging the phase separation in the P3HT:PCBM composite. For film thicknesses and electron energies corresponding to the intersection point of the two curves no material contrast of the constituents will be observed. All contrast at this voltage indicates thickness variations and composition analyses should be made at parameters away from this intersection point. The signal should be as high as possible and the distance between the two curves maximized. To minimize the influence of local thickness variations low slopes of the curves are necessary. A HAADF-STEM image of a P3HT:PCBM film is shown in Fig. 2.b. Present evaluations indicate that material contrast 
correlates partially with contrast caused by thickness variations. Extended bright regions are due to thickness variations while the composition varies at a smaller scale.

[1] J. Liu, J. Electron. Microsc. 54 (2005) 251.

[2] N.W.M. Ritchie, Surf. Interface Anal. 37 (2005) 1006.

[3] D.J. Joy et al., Scanning 11 (1989) 176.

[4] V.E. Cosslett et al., Brit. J. Appl. Phys. 15 (1964) 883.

[5] This work has been performed within the project F1 of the DFG Research Center for Functional Nanostructures (CFN). It has been further supported by a grant from the Ministry of Science, Research and the Arts of Baden-Württemberg (Az: 7713.14-300).

a)

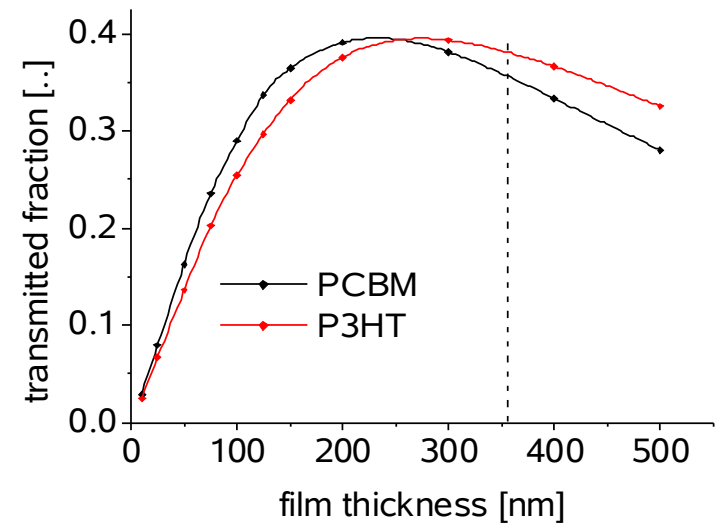

b)

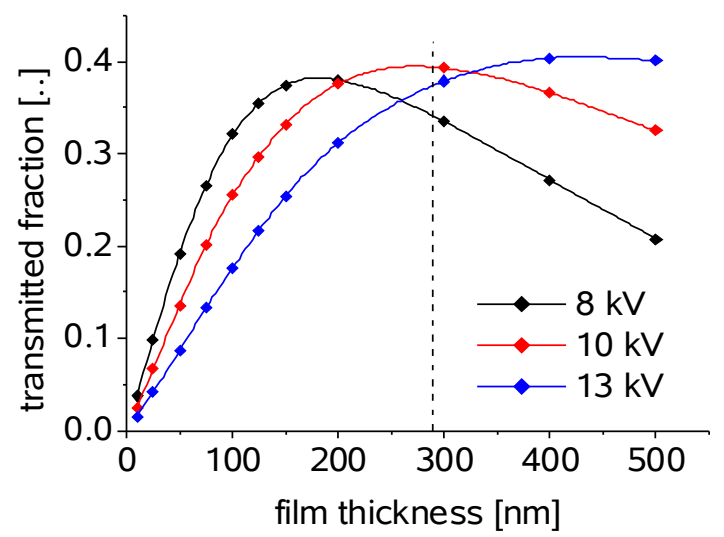

FIG. 1. (a) Monte Carlo simulation of the HAADF STEM image intensities at $10 \mathrm{keV}$ for PCBM and P3HT (b) Monte Carlo Simulation of the HAADF STEM image intensities for P3HT at different electron energies.

a)

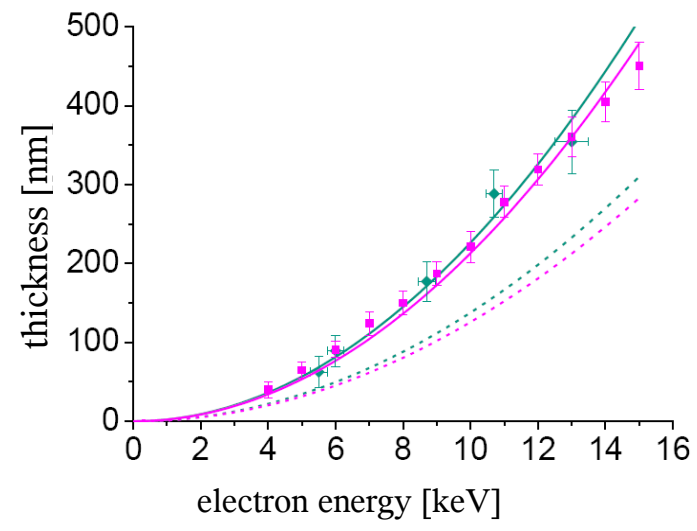

b)

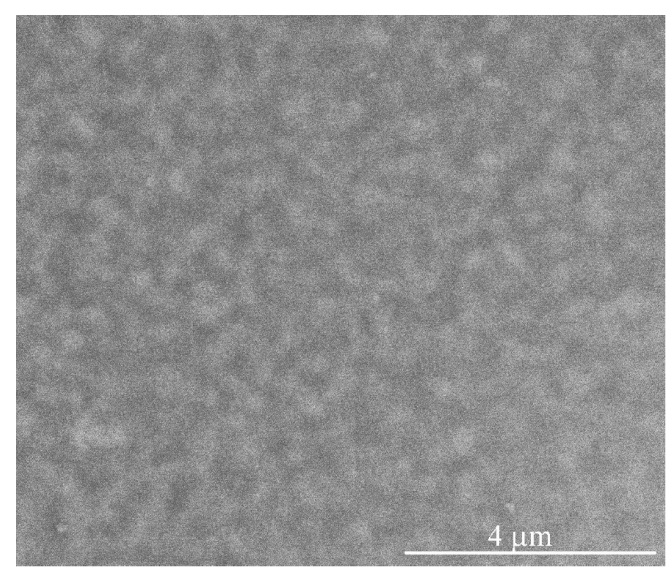

FIG. 2. (a) Maximum of transmission in P3HT:PCBM. Dependence of thickness and energy. MC simulations (upper lines), measured values (dots) and formula following Bothe [4] (lower lines) (b) STEM-HAADF image at $10 \mathrm{keV}$ of P3HT:PCBM absorber films. 